[knowledge base]

Diamond Open Access

\title{
Modules over Rings
}

\author{
Open Mathematics Collaboration*†
}

March 23, 2021

\begin{abstract}
MODULES over RINGS and its underlying definitions are presented in this white paper (knowledge base).
\end{abstract}

keywords: modules, rings, abstract algebra, knowledge base

The most updated version of this white paper is available at https://osf .io/xjsc7/download

Open Mathematics Knowledge Base http: //omkb.org

*All authors with their affiliations appear at the end of this white paper.

†Corresponding author: mplobo@uft.edu.br|Open Mathematics Collaboration 


\section{Definition}

1. Module over $R$ (left and right $R$-modules)

2. Left module over $R$ (left $R$-module)

$$
(M, R,+, \cdot)
$$

(a) $(M,+):=$ commutative group

(b) Closure under (left) scalar multiplication:

$\forall k \in R, \forall v \in M, \quad k v \in M$

(c) Scalar (left) multiplication identity: $(1 \in R, v \in M) \rightarrow(1 v=v)$

(d) Associativity of (left) scalar multiplication:

$\forall j, k \in R, v \in M, \quad(j k) v=j(k v)$

(e) (left) Distributivity of 1 scalar over 2 vectors:

$\forall k \in R, v, w \in M, \quad k(v+w)=k v+k w$

(f) (left) Distributivity of 2 scalars over 1 vector:

$\forall j, k \in R, \quad v \in M,(j+k) v=j v+k v$

$M, R:=$ sets

$(R,+, \cdot):=\operatorname{ring}$

$+, \cdot:=$ binary operations on $M, R$

$\cdot: R \times M \rightarrow M$

(scalar multiplication between elements of $R$ and $M$ )

$[1,2]$ 


\section{Right module over $R$ (right $R$-module)}

$$
(M, R,+, \cdot)
$$

(a) $(M,+):=$ commutative group

(b) Closure under (right) scalar multiplication:

$\forall k \in R, \quad \forall v \in M, v k \in M$

(c) Scalar (right) multiplication identity: $(1 \in R, v \in M) \rightarrow(v 1=v)$

(d) Associativity of (right) scalar multiplication:

$\forall j, k \in R, v \in M, v(j k)=(v j) k$

(e) (right) Distributivity of 1 scalar over 2 vectors:

$\forall k \in R, v, w \in M,(v+w) k=v k+w k$

(f) (right) Distributivity of 2 scalars over 1 vector:

$\forall j, k \in R, v \in M, v(j+k)=v j+v k$

$M, R:=$ sets

$(R,+, \cdot):=\operatorname{ring}$

$+, \cdot:=$ binary operations on $M, R$

.: $R \times M \rightarrow M$

(scalar multiplication between elements of $R$ and $M$ )

$[1,2]$ 


\section{Prerequisites}

\section{Group}

$$
(G, \star)
$$

(a) Associativity: $\forall x, y, z \in G,(x \star y) \star z=x \star(y \star z)$

(b) Identity: $\exists e \in G: \forall x \in G, e \star x=x \star e=x$

(c) Inverse: $\forall x \in G \quad \exists y \in G: x \star y=y \star x=e$

$G:=$ set

$\star:=$ binary operation

[1]

5. Commutative group (Abelian)

$$
\begin{gathered}
G_{b} \\
\forall g_{1}, g_{2} \in G_{b}, g_{1} g_{2}=g_{2} g_{1}
\end{gathered}
$$

$G_{b}:=$ group

[2]

6. Ring

$$
(R,+, \cdot)
$$
(a) $(R,+):=$ commutative group
(b) $(R, \cdot):=$ monoid
(c) Multiplication is distributive over addition in $R$
$R:=$ set
$+, \cdot:=$ binary operations on $R$ (addition and multiplication)
$[1,2]$ 
7. Binary operation

$$
\star: S \times S \rightarrow S
$$

$S:=$ set

$S \times S:=$ Cartesian product

[1]

8. Distributive

$$
\begin{array}{ll}
\forall x, y, z \in R: x \cdot(y+z)=x \cdot y+x \cdot z & \text { left distributive } \\
\forall x, y, z \in R:(y+z) \cdot x=y \cdot x+z \cdot x & \text { right distributive }
\end{array}
$$$$
[1,2]
$$

9. Semigroup

$$
\mathcal{S}=(S, \circ)
$$

$S:=$ non-empty set

$:=$ associative binary operation

[4]

10. Monoid $:=$ a semigroup $\mathcal{S}=(S, \circ)$ that contains an identity $e \in S$ such that

$$
\forall x \in S: e \circ x=x \circ e=x
$$

$[4]$

11. Associative binary operation

$$
\forall x, y, z \in S: x \circ(y \circ z)=(x \circ y) \circ z
$$

$\circ:=$ binary operation

$[4]$ 
12. Cartesian product

$$
A \times B=\{(a, b) \mid a \in A, b \in B\}
$$

$A, B:=$ sets

$A \times B:=$ Cartesian product

$(a, b):=$ ordered pair

$[3]$

13. Ordered pair

$$
(a, b)=\{\{a\},\{a, b\}\}
$$

$a:=$ first coordinate

$b:=$ second coordinate

$[1,3]$

\section{Open Invitation}

Review, add content, and co-author this white white paper $[5,6]$. Join the Open Mathematics Collaboration.

Send your contribution to mplobo@uft.edu.br.

\section{Open Science}

The latex file for this white paper together with other supplementary files are available in [7]. 


\section{Ethical conduct of research}

This original work was pre-registered under the OSF Preprints [8], please cite it accordingly [9]. This will ensure that researches are conducted with integrity and intellectual honesty at all times and by all means.

\section{Acknowledgements}

+ Center for Open Science https://cos.io

+ Open Science Framework https://osf io

\section{References}

[1] Warner, Steve. Abstract Algebra for Beginners. GET 800, 2018. https://books.google.com/books?id=UFleyAEACAAJ

[2] Dummit, David Steven, and Richard M. Foote. Abstract Algebra. Vol. 3. Hoboken: Wiley, 2004.

https://books. google.com/books?id=znzJygAACAAJ

[3] Velleman, Daniel J. How to prove it: A structured approach. Cambridge University Press, 2019.

https://books .google.com/books?vid=ISBN0521861241

[4] Cain, Alan J. Nine Chapters on the Semigroup Art. AJC Porto \& Lisbon, 2020. http://www-groups.mcs.st-and.ac.uk/alanc

[5] Lobo, Matheus P. "Microarticles." OSF Preprints, 28 Oct. 2019. https://doi.org/10.31219/osf.io/ejrct 
[6] Lobo, Matheus P. "Simple Guidelines for Authors: Open Journal of Mathematics and Physics." OSF Preprints, 15 Nov. 2019.

https://doi.org/10.31219/osf.io/fk836

[7] Lobo, Matheus P. "Open Journal of Mathematics and Physics (OJMP)." OSF, 21 Apr. 2020.

https://doi.org/10.17605/osf.io/6hzyp

https://osf .io/6hzyp/files

[8] COS. Open Science Framework. https://osf.io

[9] Lobo, Matheus P. "Modules over Rings." OSF Preprints, 15 Mar. 2021. https://doi.org/10.31219/osf.io/xjsc7

\section{The Open Mathematics Collaboration}

Matheus Pereira Lobo (lead author, mplobo@uft.edu.br) $)^{1,2}$ https://orcid.org/0000-0003-4554-1372

${ }^{1}$ Federal University of Tocantins (Brazil)

${ }^{2}$ Universidade Aberta (UAb, Portugal) 